4. David Gillman, Side approximation missing an arc, Amer. J. Math. 85 (1963), 459-476.

5. O. G. Harrold, H. C. Griffith and E. E. Posey, A characterization of tame curves in three-space, Trans. Amer. Math. Soc. 79 (1955), 12-34.

6. J. P. Hempel, Extending a surface in $E^{3}$ to a closed surface, Abstract 63T-65, Notices Amer. Math. Soc. 10 (1963), 191.

7. I. Kapuano, Sur les surfaces homeomor phes d un disque dans un $R^{2}, C$. R. Acad. Paris 256 (1953), 1229-1230.

8. E. E. Moise, Affine structures in 3-manifolds. VIII. Invariance of the knot types; local tame embedding, Ann. of Math. (2) 59 (1954), 159-170.

The Institute for Advanced Study

\title{
SPECIAL $n$-MANIFOLDS WITH BOUNDARY ${ }^{1}$
}

P. H. DOYLE AND J. G. HOCKING

By a K-R manifold we mean an $n$-manifold with boundary $M^{n}$ such that Int $M^{n}=E^{n}$ and $\mathrm{Bd} M^{n}=E^{n-1}$; Int $M^{n}$ and $\mathrm{Bd} M^{n}$ are the interior and boundary of $M^{n}$ respectively. Both Cantrell [2] and Doyle [3] have shown that for $n \neq 3$, each $K-R$ manifold is the product $E^{n-1} \times[0,1)$. But for $n=3$ there are infinitely many $\mathrm{K}-\mathrm{R}$ manifolds which are topologically distinct as pointed out in [4] and [5]. We will investigate certain properties of these manifolds with boundary.

Lemma 0. Let $M^{n}$ be a $K-R$ manifold. Then $M^{n}$ is the product $E^{n-1} \times[0,1)$ if each compact set in $M^{n}$ lies in a closed $n$-cell in $M^{n}$.

Proof. The proof is simple in that $M^{n}$ can be represented as a union of closed $n$-cells $\cup C_{i}$ where $C_{i} \cap \mathrm{Bd} M^{n}$ is an $(n-1)$-cell $D_{i}$ nicely imbedded in $\mathrm{Bd} C_{i}$ and $\mathrm{Bd} M^{n}, D_{i} \subset \operatorname{Int} D_{i+1}$ and $C_{i}-D_{i}$ CInt $C_{i+1}$, while $\left[C_{i+1}-C_{i}\right]-$ is an $n$-cell. One can then construct a homeomorphism of $M^{n}$ onto a copy of $E^{n-1} \times[0,1)$.

LEMMA 1. Let $M^{n}$ be an n-manifold with boundary. If $C$ is a compact set in $M^{n}$ such that $C \cap B d M^{n}$ lies in an open $(n-1)$-cell in $\mathrm{Bd} M^{n}$, then there is a pseudo-isotopy $h_{t}$ of $M^{n}$ onto $M^{n}$ such that $h_{1}(C) \subset F \cup C^{\prime}$, where $F$ is a fiber in a collar about $\mathrm{Bd} M^{n}$, and $C^{\prime}$ is a compact set in Int $M^{n}$.

Received by the editors August 4, 1963.

1 The work was done under National Science Foundation Grant GP-31. 
Proof. That Bd $M^{n}$ is collared in $M^{n}$ follows from [1]. Since $C \cap B d M^{n}$ lies in an open $(n-1)$-cell in $\mathrm{Bd} M^{n}$, there is a closed $n$-cell $P^{n}$ in $M^{n}$ such that $P^{n} \cap \mathrm{Bd} M^{n}$ is an $(n-1)$-cell $Q_{1}^{n-1}$, $C \cap B d M^{n} \subset$ Int $Q_{1}^{n-1}$, [Bd $\left.P^{n}-Q_{1}^{n-1}\right]-$ is an $(n-1)$-cell $Q_{2}^{n-1}$ and the set $M_{1}^{n}=\left(M^{n}-P^{n}\right) \cup Q_{2}^{n-1}$ is homeomorphic to $M^{n}$.

Since $C \cap B d M^{n} \subset$ Int $Q_{1}^{n-1}, C \cap Q_{2}^{n-1} \subset$ Int $Q_{2}^{n-1}$. Thus $C \cap P^{n}$ lies in an $n$-cell $P_{1}^{n}$ in $P^{n}, \mathrm{Bd} P_{1}^{n} \cap \mathrm{Bd} P^{n}$ is a pair of $(n-1)$-cells in Int $Q_{1}^{n-1}$ and Int $Q_{2}^{n-1}$. One can evidently find a pseudo-isotopy $h_{t}$ of $M^{n}$ onto $M^{n}$ which carries $P_{1}^{n}$ to a fiber $F$ in the collar about $\mathrm{Bd} M^{n}$, while $h_{t}$ is fixed outside any neighborhood $U$ of $P_{1}^{n}$ and for all $t, h_{t}\left(P^{n}\right)=P^{n}$. If $h_{1}$ is the terminal map, let $C^{\prime}=h_{1}\left[\left(C-P^{n}\right)\right]^{-}$. Then $h_{1}(C) \subset F \cup C^{1}$, $p=F \cap \mathrm{Bd} M^{n}$, a point.

Theorem 1. Let $M^{3}$ be a 3-dimensional $K-R$ manifold, $M^{3} \neq E^{2}$ $\times[0,1)$. Then there is a polygonal graph $G\left(G \cap \mathrm{Bd} M^{3}=p, a\right.$ point $)$ in $M^{3}$ which lies in no closed 3-cell $J^{3}$ in $M^{3}$ such that $G-p \subset$ Int $J^{3}$.

Proof. Let $M^{3}$ be given a fixed triangulation [7]. By Lemma 0 there is a compact set $C \subset M^{3}$ and $C$ lies in no closed 3-cell in $M^{3}$. We assume without loss of generality that $C \cap B d M^{3}$ is a disk $D$. Since $C$ lies in no closed 3-cell in $M^{3}, C$ lies in no closed 3-cell $K$ which meets Bd $M^{3}$ in a disk containing $D$ in its interior while $C-D C$ Int $K$.

Now by Lemma 1, $C$ can be deformed into a set of the form $h_{1}(C)$ $=F \cup C^{1}$, where $C^{1} C$ Int $M^{3}$ is compact and $F$ is a polygonal fiber in the collar about $\mathrm{Bd} M^{3}, F \cap \mathrm{Bd} M^{\boldsymbol{p}}=p$. Then again there is no closed 3-cell $K$ which meets $\mathrm{Bd} M^{3}$ in a disk containing $p$ in its interior, while $\left(F \cup C^{1}\right)-p \subset$ Int $K$. For if such a 3-cell $K$ were to exist there would be a value $0<t<1$ such that $h_{t}(C)-h_{t}(D) \subset \operatorname{Int} K$ and $h_{t}(D)$ lies interior to the disk $K \cap \mathrm{Bd} M^{3}$.

Let $N^{2}$ be the open 3-cell obtained by attaching an open collar to Bd $M^{3}$ by an extension of the triangulation on $M^{3}$. In order to construct $G$, let $H^{3}$ be a polyhedral 3-cell in Int $M^{3}$ such that $C^{1} \subset$ Int $H^{3}$ [6]. If $g_{t}$ is a pseudo-isotopy of $M^{3}$ onto $M^{3}$ which is semi-linear and fixed outside a neighborhood of $H^{3}$ in Int $M^{3}$ such that $g_{1}\left(H^{3}\right)=q$, a point, $g_{1} \mid M^{3}-H^{3}$ is a homeomorphism, then $g_{1}\left(F \cup C^{1}\right)=G$ is a polygonal graph. If there were a closed 3-cell $J^{3}$ such that Int $J^{3} \supset G-p$, one could assume that $\mathrm{Bd} J^{3}$ is locally bicollared except at $p$.

If $J_{1}^{3}$ is a 3-cell in Int $J^{3}$ except for the point $p$ of $J_{1}^{3}$ such that $G-p$ $C$ Int $J_{1}^{3}$ one can shrink $J_{1}^{3}$ to a point $p$ by a pseudo-isotopy of $M^{3}$ onto $M^{3}$ which is fixed outside of $J^{3}$. Evidently there is a closed 3-cell $K$ in $M^{3}, K \cap B d M^{s}$ is a disk with $p$ in its interior, $G-p \subset$ Int $K$. But by the construction of $G$ it follows that $F \cup C^{1}$ and hence $C$ must lie in a 3-cell. But this is contrary to hypothesis. 
One may quickly deduce from Theorem 1 the following characterization.

THEOREM 2. A necessary and sufficient condition that a 3-dimensional $K-R$ manifold $M^{3}$ be $E^{2} \times[0,1)$ is that each graph $G$ meeting $\mathrm{Bd} M^{3}$ in a point $x$ lie interior to a closed 3-cell except for $x$.

CoROllaRY. Let $M^{3}$ be a $K-R$ manifold of dimension 3 and let $p$ be a point of $\mathrm{Bd} M^{3}$. If $M^{3} \neq E^{2} \times[0,1)$, then Int $M^{3} \cup_{p}$ is not topologically the interior of a closed 3-simplex plus a point of its boundary.

Theorem 3. If $M_{1}^{3}$ and $M_{2}^{3}$ are 3-dimensional $K-R$ manifolds, then $M_{1}^{3} \times M_{2}^{3}=E^{5} \times[0,1)$ and $M_{1}^{3} \times E^{1}=E^{3} \times[0,1)$.

Proof. By either [2] or [3], a K-R manifold $M^{n}$ of dimension $n \neq 3$ is $E^{n-1} \times[0,1)$.

\section{REFERENCES}

1. M. Brown, Locally flat embeddings of topological manifolds, Topology of 3manifolds and related topics (Proc. The Univ. of Georgia Institute, 1961), pp. 83-91, Prentice-Hall, Englewood Cliffs, N. J., 1962.

2. J. C. Cantrell, Almost locally flat embeddings of $S^{n-1}$ in $S^{n}$, Bull. Amer. Math. Soc. 69 (1963), 716-718.

3. P. H. Doyle, Certain manifolds with boundary that are products, Michigan Math. J. 11 (1964), 177-181.

4. B. J. Ball, Penetration indices and applications, Topology of 3-manifolds and related topics (Proc. The Univ. of Georgia Institute, 1961), pp. 37-39, Prentice-Hall, Englewood Cliffs, N. J., 1962.

5. K. W. Kwun and F. Raymond, Manifolds which are joins, Trans. Amer. Math. Soc. 111 (1964), 108-120.

6. R. H. Bing, Approximating surfaces with poyhedral ones, Ann. of Math. (2) 61 (1957), 456-483.

7. - An alternative proof that 3-manifolds can be triangulated, Ann. of Math. (2) 69 (1959), 37-65.

Michigan State University and Virginia Polytechnic Institute 